\title{
The Prognostic Value of Optic Nerve Sheath Diameter in Patients with Poor-Grade Subarachnoid Hemorrhages
}

\author{
Dong Gyun Ju ${ }^{1}$,Jeong-Am Ryu ${ }^{1,2}$, Yong Oh Kim² ${ }^{2}$ Sangkil Lee ${ }^{3}$, Ji Sun Baek ${ }^{4}$ \\ ${ }^{1}$ Department of Neurosurgery, Samsung Medical Center, Sungkyunkwan University School of Medicine, Seoul, Korea \\ ${ }^{2}$ Department of Critical Care Medicine, Samsung Medical Center, Sungkyunkwan University School of Medicine, Seoul, Korea \\ ${ }^{3}$ Department of Neurology, ChungBuk National University Hospital, Cheongju, Korea \\ ${ }^{4}$ Department of Ophthalmology, Konyang University, Kim’s Eye Hospital, Myung-Gok Eye Research Institute, Seoul, Korea
}

Received: August 30, 2018

Accepted: September 19, 2018

Published: October 10, 2018

\section{Corresponding Author:}

Ji Sun Baek, M.D.

Department of Ophthalmology,

Konyang University, Kim's Eye

Hospital, Myung-Gok Eye

Research Institute, 136 Yeongsin-ro,

Yeongdeungpo-gu, Seoul 07260,

Korea

Tel: +82-2-2639-7812

Fax: +82-2-2633-3976

E-mail: bjs100@kimeye.com

\section{Objective}

We evaluated if the optic nerve sheath diameter (ONSD) on brain computed tomography (CT) could be used to predict neurological outcomes of patients with poor-grade subarachnoid hemorrhage (SAH).

\section{Methods}

This was a retrospective and observational study of adult patients with poor-grade SAH admitted January 2012 through June 2017. Initial brain CT was performed within 12 hours from onset of SAH. Follow-up brain CT was performed within 48 hours from onset of SAH and after aneurysmal treatment. The primary outcome was neurological status upon six months later assessed with Glasgow Outcome Scale (GOS, 1 to 5).

Results

Among 59 patients with poor-grade SAH, survival to discharge was identified in $47(79.7 \%)$ patients. Of these 47 survivors, 39 (66.1\%) had good neurological outcomes (GOS of 3, 4 or 5). In this study, initial ONSDs and follow-up ONSDs in the poor neurological outcome group were significantly greater than those in the good neurological outcome group (all $\mathrm{p}<0.03)$. The intracranial pressure (ICP) was monitored in only $13(22.0 \%)$ patients at that time of follow-CT scanning. Using simple correlation analysis, there was a linear correlation between ONSD and ICP $(\rho=0.683, p=0.010)$. In ROC curve analysis for prediction of poor neurological outcome, ONSDs and ONSD indices had considerable predictive values (C-statistics: 0.683 to 0.804 ) and similar predictive performances for poor neurological outcome.

\section{Conclusion}

The ONSDs and ONSD indices measured on CT scanning may be beneficial for predicting neurological outcomes in patients with poor-grade $\mathrm{SAH}$.

Keywords: Optic nerve sheath diameter; Brain computed tomography; Subarachnoid hemorrhage 


\section{INTRODUCTION}

Subarachnoid hemorrhage (SAH) is a complex neurovascular syndrome and a devastating condition with high mortality and morbidity rates for those that survive the initial hemorrhage $e^{2,6,8)}$. In SAH patients, the level of consciousness on admission is the most critical early predictor of clinical outcome ${ }^{2)}$. Patients with poor-grade SAH (World Federation of Neurosurgeons [WFNS] grade 4 and 5) could have high mortality and poor neurological outcome ${ }^{13)}$. However, there are recent reports on patients with poor-grade SAH treated early and aggressively with coil embolization and neurointensive care could achieve a good neurological outcome $\mathrm{e}^{6,13)}$. Therefore, to accurately evaluate the prognosis of these patients, new predictors other than the initial level of consciousness are needed.

Poor neurological outcome is usually secondary to early brain injury or delayed cerebral ischemia in SAH patients ${ }^{2}$.

Uncontrolled intracranial hypertension results in decreased cerebral perfusion and transient global cerebral ischemia ${ }^{2,5)}$.

Therefore, early monitoring of intracranial hypertension may be beneficial in predicting neurological outcomes of the patients with poor-grade $\mathrm{SAH}$.

Measurement of optic nerve sheath diameter (ONSD) has been proposed as an alternative method for detection of intracranial hypertension ${ }^{4,11)}$. However, if ONSD may be beneficial to systemically estimate neurological outcomes of patients with poor-grade SAH has not been reported. Therefore, the objective of this study is to investigate if the ONSD with some modifications could be used to predict neurological outcomes of patients with poor-grade SAH.

\section{MATERIALS AND METHODS}

\section{Study population}

This was a retrospective, single-center, and observational study of adult patients with poor-grade SAH admitted to the neurosurgical intensive care unit at Samsung Medical Center January 2012 through June 2017. This study was approved by the Institutional Review Board of Samsung Medical Center (SMC 2018-07-154). The requirement for informed consent was waived due to its retrospective nature. Clinical and laboratory data were collected by a trained study coordinator using a standardized case report form. We included patients with SAH admitted to the neurosurgical intensive care unit during the study period. Those that were obtunded or unconscious (a score of $\leq 12$ on the Glasgow Coma Scale) on admission to the hospital and those given brain computed tomography (CT) scan within 12 hours from onset of SAH were selected. Those given follow-up brain CT scan were performed after treatment of ruptured aneurysm. Of these patients, we excluded patients under age 18, those with malignancy whose expected life span was less than one year, those with insufficient medical records, those with a history of head trauma, neurosurgery, cardiac arrest, or chronic neurological abnormality on admission, and those that transferred from other hospitals after more than one day of onset of SAH. In addition, we excluded patients with anomaly of orbits, orbital mass lesions, and ocular or retro-orbital injury. A total of 59 patients with poor-grade SAH were analyzed in this study (Fig. 1).

\section{Definitions and outcomes}

In this study, poor-grade SAH was defined as World Federation of Neurosurgical Societies (WFNS) grade 4 and $5^{2}$. The primary outcome was neurological status upon six months later assessed with Glasgow Outcome Scale (GOS, 1 to 5) ${ }^{11}$. GOSs of 3, 4, and 5 were classified as good neurological outcomes. GOSs of 1 and 2 were considered poor neurological outcomes. We thoroughly reviewed medical records. Patients were graded on the GOS by two independent neurologists. Initial brain CT angiography was performed within 12 hours from onset of SAH. Follow-up brain CT was performed within 12-48 hours from onset of SAH and after aneurysmal treatment. If aneurysmal treatment was not possible, a follow-up brain CT scan was performed within 48 hours. For all CT studies, 64-channel scanners (Light Speed VCT; GE Healthcare, Milwaukee, Wisconsin, USA) with a 5-mm slice width were used. Brain $\mathrm{CT}$ images were reviewed by two independent neurologists. Investigators blinded to clinical information opened these CT scans for each patient using commercial image-viewing software (Centricity RA1000 PACS Viewer; GE Healthcare, Milwaukee, Wisconsin, USA). Optic nerve sheath diameter (ONSD) and eyeball transverse diameter (ETD) were measured using the same initial CT scan and subsequent scan. ONSD was measured at a distance of $3 \mathrm{~mm}$ behind the eyeball, immediately below the sclera in a perpendicular vector in reference to the linear axis of

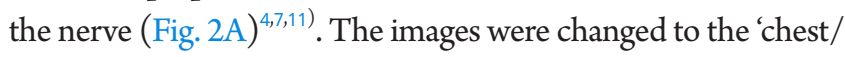
abdomen' window (window width 300 \& window level 10) and were magnified threefold on the particular image slice that demonstrated the largest diameter of the optic nerve sheath ${ }^{11)}$. ONSD was measured from one side of the optic nerve sheath to the other as a section through the center of the optic nerve ${ }^{7)}$. The transverse diameter of the eyeball was chosen because the ONSD is usually measured in the transverse plain ${ }^{15)}$. ETD was defined as the maximal transverse diameter of the eyeball measured from one side of the retina to the other (in-to-in, Fig. $2 \mathrm{~B})^{1,15)}$. The ONSD/ETD index was defined as multiplied 


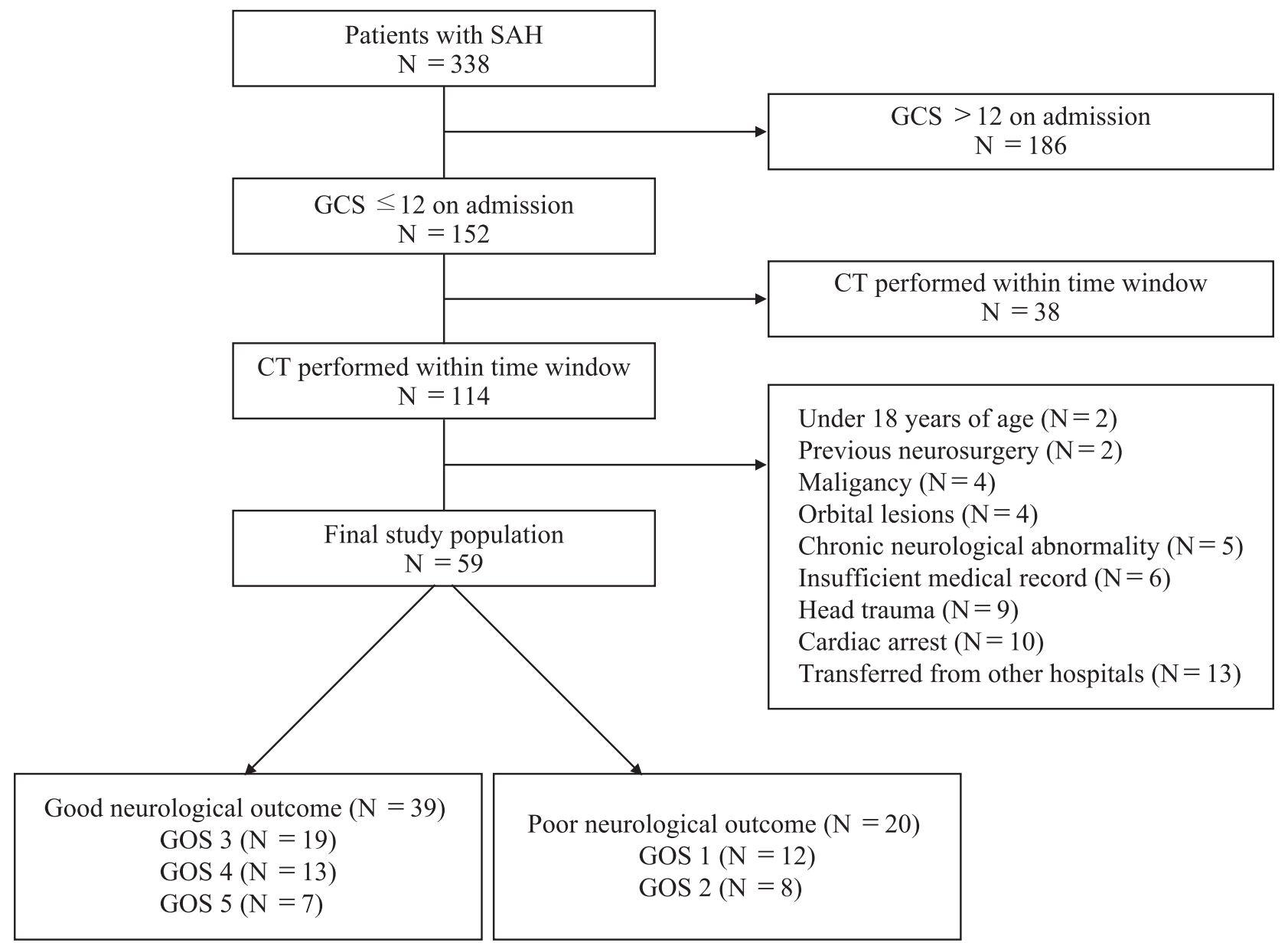

Fig. 1. Study flow chart. SAH : subarachnoid hemorrhage, GCS : Glasgow Coma Scale, CT : computed tomography, GOS : Glasgow Outcome Scale.
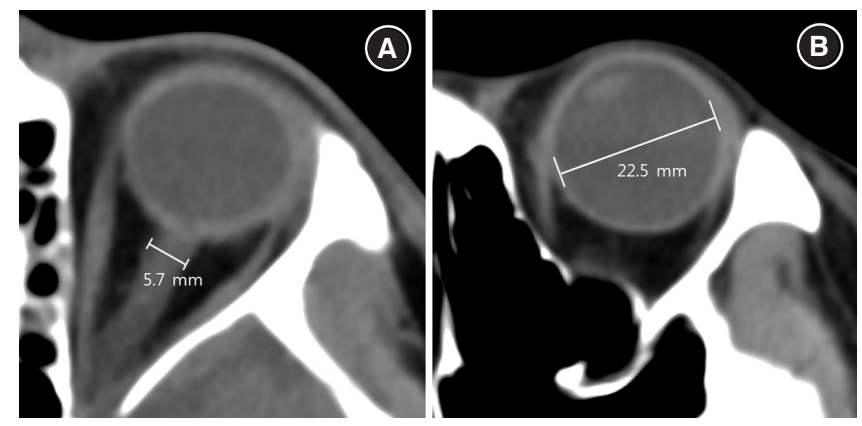

Fig. 2. A: Measurement of optic nerve sheath diameter and B : eyeball transverse diameter on the brain computed tomography scan.

median ETD $(22.5 \mathrm{~mm})$ by average value of bilateral ONSDs over average value of bilateral ETDs $\left(22.5 \times \mathrm{ONSD}_{\text {average }} /\right.$ $\mathrm{ETD}_{\text {average }}$ ). The intracranial pressure (ICP) at that time of follow-up CT scanning was defined as immediately measured ICP after the CT scanning.

\section{Statistical analyses}

All data are presented as medians and interquartile ranges (IQRs) for continuous variables and numbers (percentages) for categorical variables. Data were compared using Mann- Whitney $\mathrm{U}$ test for continuous variables and Chi-square test or Fisher's exact test for categorical variables. Simple linear regression was used to plot the relationship between simultaneously measured ONSD and ICP. Spearman's rank correlation coefficient $(\rho)$ was calculated to evaluate correlation between ONSD and ICP. Predictive performances of ONSDs and ONSD indices were assessed using area under the curve (AUC) of the receiver operating characteristic (ROC) curves for sensitivity vs. 1 -specificity. AUCs were compared using the nonparametric approach published by DeLong et al. ${ }^{3)}$ for two correlated AUCs. Optimal cut-off value of each ONSD and its modification for predicting poor neurological outcome was obtained by ROC curve and Youden index ${ }^{9,10)}$. All tests were two-sided and $\mathrm{p}$ values $<0.05$ were statistically significant. Data were analyzed using IBM SPSS statistics version 20 (IBM, Armonk, NY, USA). 


\section{RESULTS}

\section{Baseline characteristics and clinical outcomes}

The median age of patients was 64 (IQR: 53-73 years). Of 59 patients, 18 (30.5\%) were males. Hypertension (45.8\%) and smoking (18.6\%) were most common comorbidities among poor-grade SAH patients. Thirty-five (59.3\%) patients were Hunt and Hess grade 5 and 32 (54.2\%) patients were WFNS grade 5. Forty-two (71.2\%) patients had ruptured aneurysms of anterior circulation. Around middle cerebral artery (27.1\%) and anterior communicating artery $(20.3 \%)$ were most common location of ruptured aneurysm. However, aneurysm or ruptured aneurysm was not detected in four patients (6.8\%). Treatment of ruptured aneurysm was performed within 72 hours in most patients $(83.1 \%)$. Endovascular coiling of the ruptured aneurysm was performed in $36(61.0 \%)$ patients. Surgical clipping was performed in $12(20.3 \%)$ patients. Delayed cerebral ischemia accompanied in $16(27.1 \%)$ patients. Baseline characteristics of SAH patients are presented in Table 1. Survival to discharge was identified in $47(79.7 \%)$ patients. Of these 47 survivors, 39 (66.1\%) had good neurological outcomes (GOS of 3,4 or 5 , Fig. 1).

\section{Optic nerve sheath diameters and their modifications}

In this study, initial ONSDs and follow-up ONSDs in the poor neurological outcome group were significantly greater than those in the good neurological outcome group (Table 2). However, initial ETD and follow-up ETD were not significant differences between the good neurological outcome group and poor neurological outcome group $(\mathrm{p}=0.227$ and $\mathrm{p}=0.108$, respectively). The ICP was monitored in only 13 (22.0\%) patients at that time of follow-CT scanning. Using simple correlation analysis, there was linear correlation between ONSD and ICP ( $\rho=0.683, p=0.010)$ (Fig. 3). In ROC curve analysis for prediction of poor neurological outcome (Fig. 4), the C-statistic of initial $\mathrm{ONSD}_{\max }$ was 0.729 (95\% CI: 0.583 to 0.875 ). A cutoff $>6.33 \mathrm{~mm}$ had a sensitivity of $80.0 \%$ (95\% CI: $56.3 \%$ to $94.3 \%$ ) and a specificity of $69.2 \%$ (95\% CI: $52.4 \%$ to $83.0 \%$ ). The C-statistic of follow-up ONSD average was 0.760 (95\% CI: 0.633 to 0.887 ). A cut-off $>6.26 \mathrm{~mm}$ had a sensitivity of $70.0 \%$ (95\% CI: $45.7 \%$ to $88.1 \%$ ) and a specificity of $74.4 \%$ (95\% CI: $57.9 \%$ to $87.0 \%)$. The C-statistic of follow-up ONSD/ETD was 0.804 (95\% CI: 0.684 to 0.924 ). A cut-off $>6.46$ had a sensitivity of $65.0 \%$ (95\% CI: $40.8 \%$ to $84.6 \%$ ) and a specificity of $87.2 \%$ ( $95 \% \mathrm{CI}: 72.6 \%$ to $98.7 \%$ ). However, there were similar predictive performances of ONSDs and ONSD indices for poor neurological outcome; the predictive performance of follow-up ONSD/ETD for poor neurological outcome was not

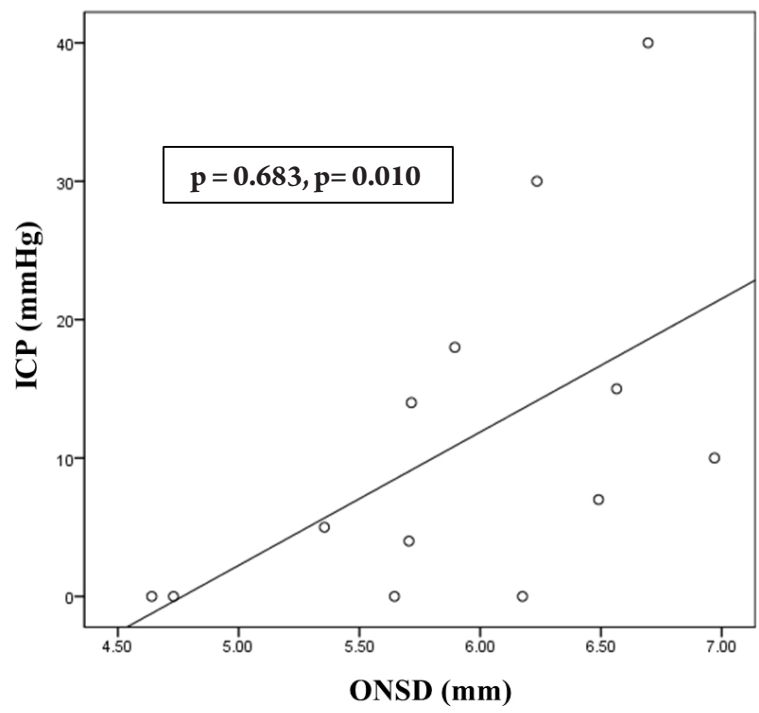

Fig. 3. Scatter plot of intracranial pressure (ICP, $\mathrm{mmHg}$ ) and optic nerve sheath diameter (ONSD, mm). Follow-up $\mathrm{ONSD}_{\text {average }}$ was used in simple correlation analysis.

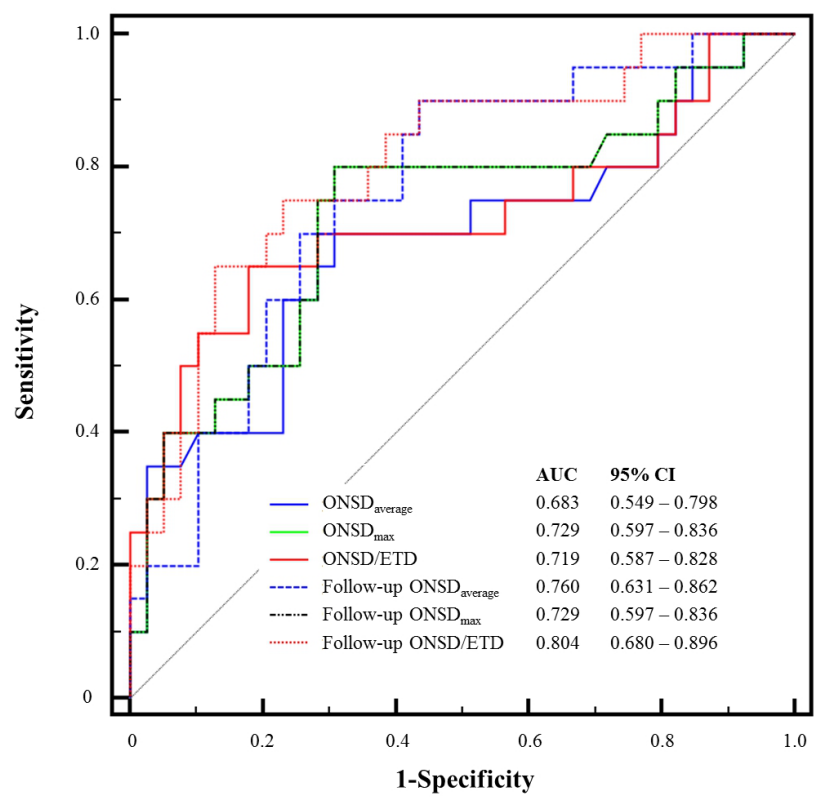

Fig. 4. Receiver operating characteristic (ROC) curves for the prediction of poor outcomes using the optic nerve sheath diameters and their modifications. ONSD : optic nerve sheath diameter, ETD : eyeball transverse diameter.

significantly different compared to that of initial $\mathrm{ONSD}_{\max }$ or follow-up $\mathrm{ONSD}_{\text {average }}(\mathrm{p}=0.199$ and $\mathrm{p}=0.156$, respectively $)$.

\section{DISCUSSION}

In this study, we evaluated if the ONSDs and their modifications could be used to predict neurological outcomes of patients 
Table 1. Baseline characteristics

\begin{tabular}{|c|c|c|c|}
\hline & $\begin{array}{l}\text { Good neurological outcome } \\
\qquad(n=39)\end{array}$ & $\begin{array}{l}\text { Poor neurological outcome } \\
\qquad(\mathbf{n}=20)\end{array}$ & p-value \\
\hline Age (yr) - median (IQR) & $58.0(49.0-68.0)$ & $72.0(61.5-76.5)$ & 0.005 \\
\hline Gender, male — no. of patients (\%) & $11(28.2)$ & $7(35.0)$ & 0.812 \\
\hline $\mathrm{BMI}\left(\mathrm{kg} / \mathrm{m}^{2}\right)-\operatorname{median}(\mathrm{IQR})$ & $23.4(21.1-26.5)$ & $22.0(20.2-24.0)$ & 0.206 \\
\hline \multicolumn{4}{|l|}{ Comorbidities — no. of patients (\%) } \\
\hline Hypertension & $20(51.3)$ & $7(35.0)$ & 0.362 \\
\hline Diabetes mellitus & $3(7.7)$ & $4(20.0)$ & 0.338 \\
\hline Previous stroke & $3(7.7)$ & $0(0.0)$ & 0.518 \\
\hline Hunt \& Hess Classification — no. of patients (\%) & & & 0.002 \\
\hline 4 & $22(56.4)$ & $2(10.0)$ & \\
\hline 5 & $17(43.6)$ & $18(90.0)$ & \\
\hline Fisher classification — median (IQR) & $4.0(3.0-4.0)$ & $4.0(4.0-4.0)$ & 0.010 \\
\hline Modified Fisher classification — median (IQR) & $3.0(3.0-4.0)$ & $4.0(3.5-4.0)$ & 0.016 \\
\hline Glasgow Coma Scale — median (IQR) & $7.0(4.0-8.0)$ & $4.0(3.0-6.5)$ & 0.010 \\
\hline Pupil reactivity — no. of patients (\%) & & & 0.012 \\
\hline Both intact pupil reflex & $27(69.2)$ & $6(30.0)$ & \\
\hline One unreactive pupil & $3(7.7)$ & $2(10.0)$ & \\
\hline Both unreactive pupil & $9(23.1)$ & $12(60.0)$ & \\
\hline \multicolumn{4}{|l|}{ Aneurysm location — no. of patients (\%) } \\
\hline Anterior communicating artery & $8(20.5)$ & $4(20.0)$ & \\
\hline Anterior cerebral artery \& distal & $5(12.8)$ & $2(10.0)$ & \\
\hline Hydrocephalus - no. of patients (\%) & $23(59.0)$ & $16(80.0)$ & 0.185 \\
\hline Intraventricular hemorrhage - no. of patients (\%) & $18(46.2)$ & $15(75.0)$ & 0.066 \\
\hline EVD — no. of patients (\%) & $32(82.1)$ & $8(40.0)$ & 0.003 \\
\hline Vasospasm — no. of patients (\%) & $20(51.3)$ & $4(20.2)$ & 0.021 \\
\hline Delayed cerebral ischemia — no. of patients (\%) & $13(33.3)$ & $3(15.0)$ & 0.134 \\
\hline Early treatment within $72 \mathrm{hr}-$ no. of patients (\%) & $39(100.0)$ & $10(50.0)$ & $<0.001$ \\
\hline \multicolumn{4}{|l|}{ Aneurysm management — no. of patients (\%) } \\
\hline Coiling & $27(69.2)$ & $9(45.0)$ & 0.127 \\
\hline Clip operation & $10(25.6)$ & $2(10.0)$ & 0.284 \\
\hline Endotracheal intubation during over $24 \mathrm{hr}$ & $23(59.0)$ & $20(100.0)$ & 0.002 \\
\hline Decompressive craniectomy — no. of patients (\%) & $5(12.8)$ & $3(15.0)$ & 0.999 \\
\hline Barbiturate coma therapy — no. of patients (\%) & $2(5.1)$ & $3(15.0)$ & 0.427 \\
\hline
\end{tabular}

IQR : interquartile range, BMI : body mass index, WFNS : World Federation of Neurosurgeons, EVD : external ventricular drainage

with poor-grade SAH. Major findings of this study were as follows: 1) the patients with poor-grade $\mathrm{SAH}$ had considerable survival rate $(79.7 \%)$ and favorable neurological prognosis (66.1\%); 2) Simultaneous measurement of ONSD on CT and
ICP were moderately correlated; 3 ) In initial CT and followup CT, ONSDs and ONSD indices of the poor neurological outcome group were significantly greater than those of the good neurological outcome group and these markers could be used to 
Table 2. The optic nerve sheath diameters and their modifications according to neurological outcomes

\begin{tabular}{lccc}
\hline & Good neurological outcome $(\mathbf{n}=\mathbf{3 9})$ & Poor neurological outcome $(\mathbf{n}=\mathbf{2 0})$ & p-value \\
ONSD $_{\text {average }}$ & $6.07(5.87-6.47)$ & $6.62(5.98-7.07)$ & 0.022 \\
ONSD $_{\text {max }}$ & $6.21(5.93-6.71)$ & $6.79(6.39-7.36)$ & 0.004 \\
$\mathrm{ETD}_{\text {average }}$ & $22.7(22.0-23.3)$ & $22.3(21.8-22.9)$ & 0.227 \\
$\mathrm{ONSD}_{\text {ETD }}$ & $6.13(5.86-6.32)$ & $6.81(5.99-7.11)$ & 0.006 \\
Follow-up ONSD & $5.81(5.45-6.29)$ & $6.52(6.11-6.69)$ & 0.001 \\
Follow-up ONSD & $6.21(5.93-6.71)$ & $6.79(6.39-7.36)$ & 0.004 \\
Follow-up ETD & $22.7(22.1-23.1)$ & $6.63(6.21-6.84)$ & 0.108 \\
Follow-up ONSD/ETD & $5.85(5.39-6.26)$ & $<0.001$ & \\
\hline
\end{tabular}

ONSD : optic nerve sheath diameter, ETD : eyeball transverse diameter

predict neurological outcomes of patients with poor-grade SAH. Although it is difficult to predict the outcome following SAH, level of consciousness on admission would be associated with prognosis of SAH patients ${ }^{2,6,13)}$. One widely accepted predictor is the WFNS grade based on the level of consciousness as assessed by the Glasgow coma scale ${ }^{13,14)}$. Especially, patients with poor WFNS grade SAH 4 and 5 historically have had high mortality and poor neurological outcomes ${ }^{13)}$. However, in recent years, a considerable number of patients with poor-grade $\mathrm{SAH}$ treated aggressively with early coil embolization and supportive neurointensive care could achieve a favorable neurological outcome and survival ${ }^{6,13)}$. Therefore, to accurately evaluate the prognosis of patients with poor-grade SAH, other predictors other than the initial level of consciousness are needed.

In patients with poor-grade $\mathrm{SAH}$, poor neurological outcome is usually secondary to early brain injury or delayed cerebral ischemia ${ }^{2}$. Secondary cerebral injury can occur, that is the additive cerebral injury characterized by an imbalance in postresuscitation cerebral oxygen delivery and use, ultimately culminating in neuronal death ${ }^{5}$. In addition, cerebral autoregulation may be disturbed to different extents and disturbed autoregulation in SAH is associated with delayed cerebral ischemia, vasospasm and unfavorable outcome ${ }^{5}$. Especially, uncontrolled intracranial hypertension results in decreased cerebral blood flow and transient global cerebral ischemia and associated with early brain injury and poor neurological outcome $e^{2,5)}$. Therefore, early monitoring of intracranial hypertension may be beneficial in predicting neurological outcomes of the patients with poor-grade SAH.

Measurement of ONSD has been proposed as an alternative method for the detection of increased intracranial pressure ${ }^{4)}$. The optic nerve is surrounded by cerebrospinal fluid because it is a part of the central nervous system. Therefore, increased intracranial pressure will be transmitted through the subarachnoid space surrounding the optic nerve, within the nerve sheath, especially the retrobulbar segment, unless circulation of cerebrospinal fluid is not blocked ${ }^{4}$. ONSD on initial brain CT may be correlated with neurologic outcomes after traumatic brain injury ${ }^{11,12)}$. Simultaneous measurement of ONSD on initial CT and intracranial pressure were correlated, and ONSD was indicative of intracranial hypertension in patients with severe traumatic brain injury ${ }^{11,12)}$. In this study, ONSD measured on CT scanning may be also a good predictive marker associated with intracranial pressure and prognosis in patient with poor-grade $\mathrm{SAH}$.

This study has several limitations. First, it was a retrospective review of medical records. Therefore, the GOS was also retrospectively determined based on medical records. Second, the non-randomized nature of the registry data may have resulted in selection bias. Although brain CT scans were performed within 48 hours after SAH, a major limitation of this study may be that the CT scans were performed at different time settings. Third, invasive ICP monitoring was performed in a limited number of patients after aneurysmal treatment. Finally, our study was conducted at one institution and involved a lesser number of subjects. Although there were no significant differences between ONSDs and their modifications in this study, there may be different outcomes in larger cohorts. Therefore, future studies with larger cohorts are needed to validate these findings.

\section{CONCLUSION}

The ONSDs and ONSD indices measured on CT scanning may be used to predict neurological outcomes of patients with poor-grade SAH.

\section{CONFLICT OF INTEREST}

No potential conflict of interest relevant to this article was reported. 


\section{INFORMED CONSENT}

Informed consent was obtained from all individual participants included in this study.

\section{REFERENCES}

1. Bekerman I, Sigal T, Kimiagar I, Ben Ely A, Vaiman M. The quantitative evaluation of intracranial pressure by optic nerve sheath diameter/eye diameter CT measurement. Am J Emerg Med 2016;34:2336-2342.

2. de Oliveira Manoel AL, Goffi A, Marotta TR, Schweizer TA, Abrahamson S, Macdonald RL. The critical care management of poor-grade subarachnoid haemorrhage. Crit Care 2016;20:21.

3. DeLong ER, DeLong DM, Clarke-Pearson DL. Comparing the areas under two or more correlated receiver operating characteristic curves: a nonparametric approach. Biometrics 1988;44:837-845.

4. Hwan Kim Y, Ho Lee J, Kun Hong C, Won Cho K, Hoon Yeo J, Ju Kang M, et al. Feasibility of optic nerve sheath diameter measured on initial brain computed tomography as an early neurologic outcome predictor after cardiac arrest. Acad Emerg Med 2014;21:1121- 1128.

5. Johnson U, Engquist H, Lewen A, Howells T, Nilsson P, Ronne-Engstrom E, et al. Increased risk of critical CBF levels in SAH patients with actual CPP below calculated optimal CPP. Acta Neurochir (Wien) 2017;159:1065-1071.

6. Konczalla J, Seifert V, Beck J, Guresir E, Vatter H, Raabe A, et al. Outcome after Hunt and Hess Grade V subarachnoid hemorrhage: a comparison of pre-coiling era (19801995) versus post-ISAT era (2005-2014). J Neurosurg 2018;128:100-110.

7. Legrand A, Jeanjean P, Delanghe F, Peltier J, Lecat B, Dupont H. Estimation of optic nerve sheath diameter on an initial brain computed tomography scan can contribute prognostic information in traumatic brain injury patients. Crit Care
2013;17:R61.

8. Naval NS, Chang T, Caserta F, Kowalski RG, Carhuapoma JR, Tamargo RJ. Improved aneurysmal subarachnoid hemorrhage outcomes: a comparison of 2 decades at an academic center. J Crit Care 2013;28:182-188.

9. Ruopp MD, Perkins NJ, Whitcomb BW, Schisterman EF. Youden Index and optimal cut-point estimated from observations affected by a lower limit of detection. Biom J 50:2008;419-430.

10. Schisterman EF, Perkins NJ, Liu A, Bondell H. Optimal cutpoint and its corresponding Youden Index to discriminate individuals using pooled blood samples. Epidemiology $16: 2005 ; 73-81$.

11. Sekhon MS, Griesdale DE, Robba C, McGlashan N, Needham E, Walland K, et al. Optic nerve sheath diameter on computed tomography is correlated with simultaneously measured intracranial pressure in patients with severe traumatic brain injury. Intensive Care Med 40:2014;12671274.

12. Sekhon MS, McBeth P, Zou J, Qiao L, Kolmodin L, Henderson WR, et al. Association between optic nerve sheath diameter and mortality in patients with severe traumatic brain injury. Neurocrit Care 2014;21:245-252.

13. Taylor CJ, Robertson F, Brealey D, O'Shea F, Stephen T, Brew $S$, et al. Outcome in poor grade subarachnoid hemorrhage patients treated with acute endovascular coiling of aneurysms and aggressive intensive care. Neurocrit Care 2011;14:341347.

14. Teasdale GM, Drake CG, Hunt W, Kassell N, Sano K, Pertuiset B, et al. A universal subarachnoid hemorrhage scale: report of a committee of the World Federation of Neurosurgical Societies. J Neurol Neurosurg Psychiatry 1988;51:1457.

15. Vaiman M, Gottlieb P, Bekerman I. Quantitative relations between the eyeball, the optic nerve, and the optic canal important for intracranial pressure monitoring. Head Face Med 2014;10:32. 